\title{
APROXIMACIÓN AL FENÓMENO DE LA INTEGRACIÓN GANADERA EN LA COMUNIDAD VALENCIANA
}

\author{
José Antonio Segrelles Serrano
}

\begin{abstract}
RESUMEN
Las profundas mutaciones socio-económicas que experimenta España desde finales de la década de los años cincuenta no sólo provoca la intensificación de los métodos productivos en la ganadería sino que, asimismo, aparecen en el sector agropecuario nuevas relaciones funcionales, económicas y sociales. La integración vertical ha establecido diferentes puntos de contacto entre los diversos elementos que componen la cadena productiva y crea una estructura desequilibrada con indudables consecuencias negativas para la explotación familiar.
\end{abstract}

\begin{abstract}
Since the last 50's, deep social and economic changes happen in Spain, which promoted the intensification of the production methods in cattle-raising. At the same time new functional, economical and social relationships appear in this area and in farming. The straight integration stablishes several conections between different elements in the production chain and creates an unbalanced structure that disfavour the familiar exploitations.
\end{abstract}

Los cambios socio-económicos experimentados por España desde finales de la década de los años cincuenta y comienzo de los sesenta repercuten directamente en la actividad pecuaria debido a la ingente demanda de productos ganaderos solicitada por la población. Ello provoca la intensificación de los métodos productivos, mutaciones en la composición interna de la cabaña y una acusada modificación de las áreas pecuarias. A esta situación no se accede por la evolución de la empresa agraria, sino que el origen radica en inversiones capitalistas que previamente penetran en el sector al tiempo que también lo hacen las nuevas tecnologías. Con la combinación de estos elementos surgen en el medio rural relaciones funcionales, sociales y económicas de nuevo cuño que perturban el antiguo orden secular y alteran los modos tradicionales.

Actualmente, podemos distinguir, grosso modo, tres sistemas de explotación pecuaria intensiva: la empresa libre, el trabajo cooperativo y la integración vertical. La explotación directa, personal e independiente tiene cada vez menos protagonismo, pues las duras y 
críticas condiciones económicas actuales sólo dejan sobrevivir a los más fuertes y supeditan la empresa libre a los dictámenes de una estructura oligopolística que domina la producción. El futuro de la explotación familiar en este contexto es muy sombrío. Por otro lado, el trabajo cooperativo está muy poco extendido en España si lo comparamos con los demás países de la $\mathrm{CEE}$, aunque existen pujantes cooperativas que atesoran notables cuotas de mercado. Son, sin embargo, ciertas firmas, las más de las veces con capital multinacional, las que dominan todos los resortes de la producción ganadera intensiva (genética, sanidad, cereales, oleaginosas, piensos compuestos, mataderos, transportes, comercialización...). Estas empresas han contribuido enormemente a modificar los métodos de explotación, comercio y transformación mediante el empleo de fórmulas de integración vertical, cuyo fin prioritario es el control absoluto de la cadena productiva (amont y aval). Hoy en día concentran un gran poder económico que les permite especular con los precios, producciones y mercados, quedando los ganaderos familiares e independientes a su merced.

Por lo que respecta a la producción pecuaria stricto sensu, el fenómeno de la integración consiste básicamente en la cría por cuenta ajeno de determinadas especies animales, sobre todo porcino y pollos de carne que son los que mejor transforman los piensos compuestos en carne. Esto supone el establecimiento de nuevas relaciones entre los productores, los suministradores de input y los que transforman y comercializan la producción. Los engranajes del mecanismo integrador dan lugar al neto predominio del capital y a un imparable cambio en la propiedad de los medios de producción que configura, en la actualidad, un panorama de concluyentes repercusiones económicas y sociales.

\section{Nacimiento y evolución de la integración ganadera}

A finales de los años cincuenta se introducen en España las primeras estirpes de aves americanas a través de ciertas áreas entre las que destaca Cataluña, región de afamada tradición avícola. Los pioneros europeos fueron, casi una década antes, los Países Bajos y Bélgica ${ }^{1}$. Estos híbridos avícolas se obtienen mediante sofisticados programas genéticos y para realizar con eficacia su función productiva precisan una alimentación específica a base de concentrados que se elaboran con materias primas y correctores importados de Estados Unidos. Las importaciones de tecnología, cereales y oleaginosas fueron impulsadas por la Orden de 20 de junio de 1960 (BOE 7-julio-1960), que reducía los aranceles de las compras de productos agropecuarios en el exterior.

La demanda poblacional de productos cárnicos y la oferta de los medios idóneos para satisfacerla (piensos y genética) se conjugan para ofrecer un sistema industrializado de explotación pecuaria que tiene su embrión en las fábricas de piensos compuestos. Éstas se desarrollan también a comienzos de los años sesenta, ya que antes de dichas fechas los piensos se producían de modo artesanal en las propias explotaciones ganaderas. La producción industrializada de concentrados es consustancial a los nuevos métodos y se singulariza por un acusado poder económico y por una fuerte dependencia financiera y tecnológica del exterior. Es el capital extranjero el principal impulsor de esta industria, incluso

\footnotetext{
${ }^{1}$ DIRY, J. P., L'industrialisation de l'elevage en France. (Economie et géographie des filières avicoles et porcines), Éditions Ophrys, 1985, pp. 179-180.
} 
son foráneas las sociedades importadoras de materias primas y las molturadoras de soja (v. gr. Continental Grain, Cargill, Bunge.... $)^{2}$.

La inmediata producción masiva de carne de ave que deriva de estos métodos revolucionarios provoca a lo largo de la década de los años sesenta una serie de crisis periódicas de sobreproducción porque el mercado no puede absorber semejante oferta. Las lógicas repercusiones de estas crisis hicieron estragos entre las explotaciones menos desarrolladas, pero favorecieron a las empresas capitalizadas y con ajustados costes de producción. Asimismo, los volúmenes de concentrados producidos obliga a las fábricas de piensos a asegurarse la venta y cobro de sus productos. De esta forma, las firmas consagradas a los piensos compuestos se convierten en «creadoras» de ganadería intensiva ${ }^{3}$, expanden este tipo de aprovechamiento pecuario por ser el que mejor convierte los alimentos concentrados en carne. El mecanismo utilizado para ello es el de la integración, pues así encuentran destinatario a sus elaborados, garantizan el cobro de los mismos en ganado vivo y consiguen los beneficios añadidos que genera la comercialización y transformación de los animales. El polo difusor de la integración vertical española reside en las fábricas de piensos, construyendo relaciones contractuales amont con los ganaderos. Algunos trabajos sobre el tema estiman que el control de la integración vertical en Cataluña lo ejercen los suministradores de piensos en un $90 \%{ }^{4}$.

Hasta mediados de los años setenta las fábricas de piensos fomentan la expansión ganadera financiando las instalaciones a unos granjeros que todavía eran independientes y asumían los riesgos del mercado. A raíz de las sucesivas quiebra de los precios y de los exiguos márgenes que deja el sector, muchos avicultores abandonan la producción, pero otros se integran en las cadenas productivas de las grandes empresas para poder subsistir. Sin embargo, el número de granjeros libres aún es importante porque las expectativas amortizadoras de las inversiones en instalaciones se mostraban todavía permisivas. Después de la denominada crisis del petróleo la situación cambia. Los efectos de la crisis provocan otra notable oleada de abandonos, sobre todo por lo que respecta a las explotaciones menos profesionalizadas. El precio de los input crece vertiginosamente mientras que los de la carne no van a la par por sus desmesuradas ofertas y carácter intensivo. Las inversiones son más costosas y el progresivo endeudamiento hace peligrar la estabilidad económica de las pequeñas y medianas empresas. Asimismo, las masivas concentraciones de animales en espacios reducidos son excelente caldo de cultivo para la aparición de devastadoras enfermedades. Todo ello desalienta al ganadero independiente y fortalece a unas firmas boyantes que cada vez dominan más los precios y los mercados.

Las explotaciones porcinas acusan más si cabe las oscilaciones de los precios por su marcado carácter cíclico ${ }^{5}$. Los vaivenes coyunturales afectan a las empresas poco capitalizadas, con escasos recursos económicos. Si a ello unimos la virulencia de los frecuentes

\footnotetext{
${ }^{2}$ LANGREO NAVARRO, A., «Análisis de la integración vertical en España», Agricultura y Sociedad, 9, octubrediciembre 1978, pp. 193-194.

${ }^{3}$ BUXADE CARBO, C., Perspectivas de la ganadería española, Madrid, Instituto de Empresa, 1982, p. 243.

${ }^{4}$ ALDOMA BUIXADE, J., VILLARREAL MORENO, J., VIÑAS FOLCH, Ll., La integració en la Ramadería a Catalunya, Barcelona, Departament d'Agricultura, Ramaderia i Pesca, Generalitat de Catalunya, 1983, p. 37.

${ }^{5}$ WIENBERG, D., SOBRINO, F., El ciclo del cerdo en España, Madrid, Departamento de Economía Agraria, C.S.I.C., 1958, 108 pp.
} 
brotes de Peste Porcina Africana se pueden comprender los riesgos que acechan la producción porcina por cuenta propia. La indefensión del pequeño y mediano empresario es total cuando se recrudecen las epizootías y/o caen los precios después de haber inmovilizado su capital (obtenido muchas veces a través de créditos) en las cuantiosas inversiones que conllevan las instalaciones modernas. El intento de paliar estos peligros es lo que hace prosperar el fenómeno de la ganadería contractual. Ahora ya no son las empresas integradoras las que buscan la captación de nuevos integrados, sino que son los mismos ganaderos, acuciados por las dificultades, los que solicitan la inclusión en el seno de poderosas firmas agro-alimentarias para evitar así los riesgos que asolan este tipo de ganadería y poder permanecer en el sector a la vez que obtienen interesantes rentas complementarias, y ello aun a costa del beneficio y del poder de decisión sobre su empresa.

De todos modos, la integración vertical alcanza su punto culminante cuando al suministro de piensos se añade la asesoría técnica y la asistencia veterinaria y cuando se cierra el ciclo mediante relaciones contractuales con las industrias cárnicas. Una vez controlados los input y el cebo de los animales el siguiente paso consiste en dominar la transformación y la distribución. La relación de la fábrica de piensos con la industria transformadora ( $a v a l$ ) puede ser de capital, contractual o de simple alquiler. Hoy en día la necesidad imperiosa de sacrificar los animales en cuanto alcanzan el peso óptimo, las fuertes inversiones que precisan los modernos mataderos frigoríficos e industriales y su vinculación directa a los circuitos comerciales, han desplazado muchas veces el núcleo de la cadena integradora en detrimento de la fábrica de piensos. Sin embargo, aunque el matadero cierre el ciclo productivo y adquiera papel decisorio, en raras ocasiones se inicia en él el proceso integrador, al contrario de lo que sucede en varios países europeos como por ejemplo Gran Bretaña ${ }^{6}$.

Hasta los primeros años de la década de los ochenta continúa la acción absorbente de las integradoras y el fenómeno se consolida como sistema de explotación casi exclusivo. A partir de aquí el proceso integrador se estabiliza, pero el aprovechamiento pecuario intensivo ya se concentra en unas pocas manos que conceden escasos márgenes a otras iniciativas. Aunque se trata de un modelo perfectamente asimilado y extendido en Europa Occidental ${ }^{7}$, la integración española es especialmente intensa. La avicultura híbrida de aptitud cárnica estaba integrada hacia 1970 en un $40 \%$ del total producido, mientras que en la actualidad supera el 95\%, llegando al $100 \%$ en algunos sectores catalanes, aragoneses y valencianos según revelan las encuestas orales. El ganado de cerda se halla más o menos integrado en función de su orientación productiva. Las explotaciones de cebo oscilan entre el 60 y el 70\%; la producción de lechones apenas llega al $20 \%$. La menor integración de la cría se debe a sus vínculos con la explotación familiar, ligada a la agricultura en notable medida, y a la exigencia de trabajo especializado. El engorde no requiere grandes conocimientos técnicos y ha sido cultivado por improvisados ganaderos que ante las perspectivas de una sustanciosa rentabilidad no dudaron en formar parte de la expansión desencadenada por las integradoras en áreas de escasa tradición pecuaria.

Los intentos de criar terneros para carne y corderos precoces no han tenido demasiado éxito por lo mal que transforman los piensos compuestos y por el rígido soporte financiero que reclaman. El grado de integración es poco significativo aunque en los

\footnotetext{
${ }^{6}$ SOBRINO IGUALADOR, F., y OTROS, «Evolución de los sistemas ganaderos en España», Revista de Estudios Agro-Sociales, 116, julio-septiembre 1981, p. 83.

${ }^{7}$ SCEAU, R., «La coopération agricole dans la région Rhone-Alpes», Revue de Geographie de Lyon, 1972, p. 285.
} 


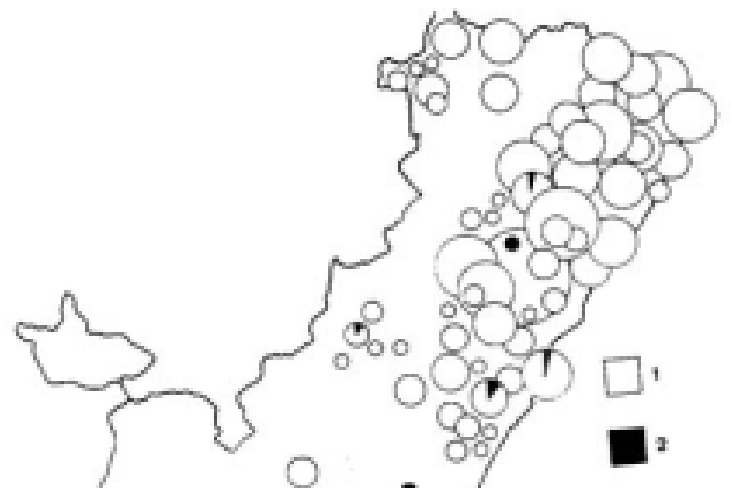

FIGURA 1. Comunidad Valenciana. Distribución municipal de las explotaciones avícolas de carne. Primer

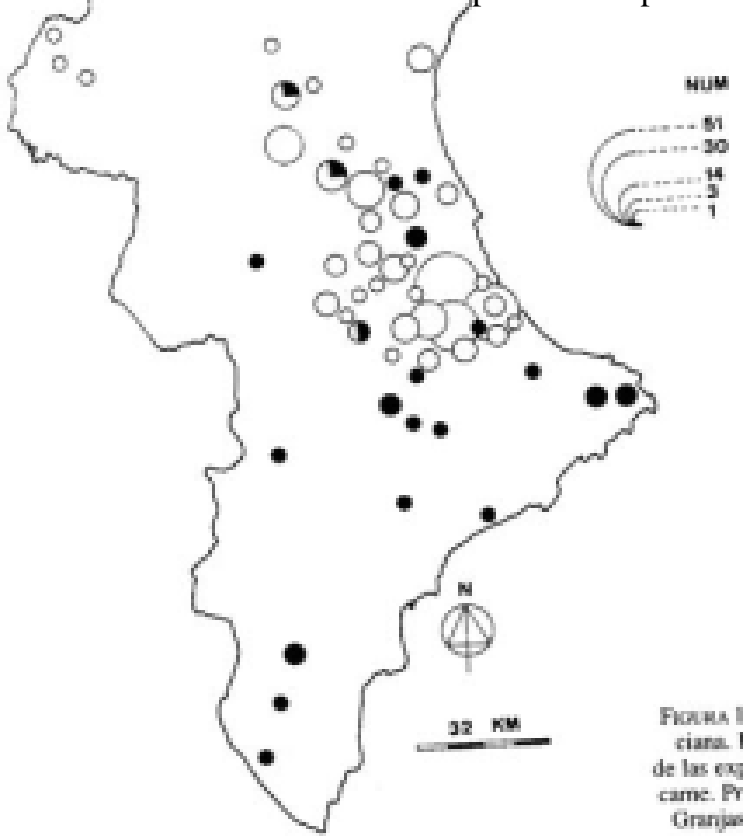


semestre 1988. 1, Granjas integradas; 2, Granjas independientes. 


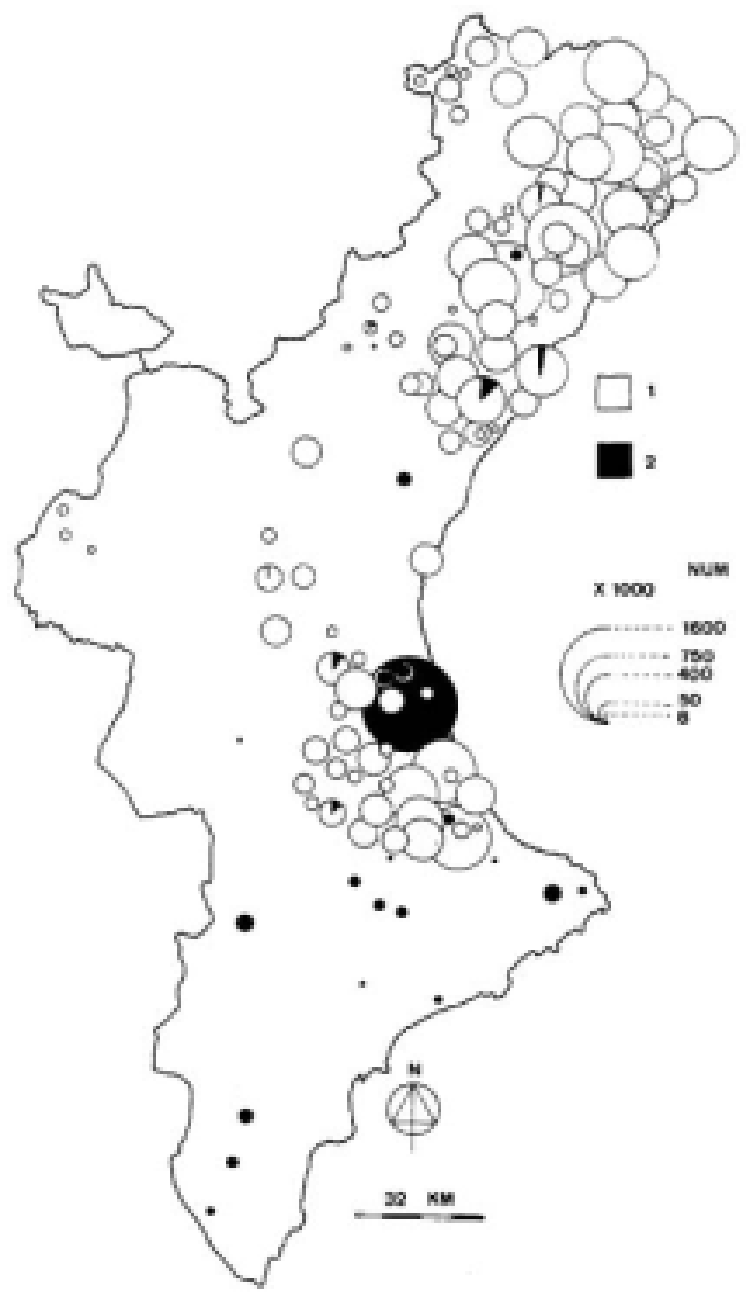

FIGURA 2. Comunidad Valenciana. Distribución municipal del censo avícola de carne. Primer semestre 1988. 1, Granjas integradas; 2, Granjas independientes. 
tiempos más recientes esté creciendo la integración bovina. Al comenzar los años ochenta se encuentra integrada el $25-30 \%$ de la producción catalana de terneros de aptitud cárnica ${ }^{8}$.

Los pavos criados con sistemas intensivos son de reciente aparición. Se hallan integrados en un $100 \%$.

\section{La integración ganadera valenciana y su reparto territorial}

Cataluña es la región pionera en la intensificación pecuaria. Aquí se localizaron los primeros avances genéticos y de alimentación animal que penetraron en España, así como las primeras multinacionales. El desarrollo económico y socio-demográfico, la expansión del mercado barcelonés, la presencia de activas y capaces instalaciones portuarias, la densa red de carreteras y ferrocarriles y el carácter emprendedor del empresario catalán, constituyeron un complejo foco de atracción para los nuevos modos productivos. Como consecuencia de todo ello creció y se desarrolló el fenómeno integrador. A partir del efecto difusor de Cataluña, la industrialización pecuaria penetró en la Comunidad Valenciana, región donde encontró las condiciones físicas, agrarias, económicas y demográficas idóneas para su desarrollo. Asimismo, la intensificación productiva supuso el auge de las fórmulas contractuales en la explotación del ganado porcino y de la avicultura de carne. El grado de integración avícola es muy similar al catalán, es decir, por encima del 90-95\%, porcentajes en los que coinciden los técnicos entrevistados de ambas Comunidades Autónomas. La integración porcina de cebo es inferior a la de Cataluña, pues mientras en Lérida y Tarragona los valores se hallan sobre el $85 \%$ del total, la Comunidad Valenciana ofrece cifras más modestas. Según el Registro de Explotaciones Porcinas de la Conselleria d'Agricultura de la Generalitat Valenciana de las 469.664 plazas ganaderas existentes, tanto en producción como en cebo, se hallan integradas 267.774 , es decir, el $57 \%$. Si tenemos en consideración las opiniones que sostienen que la cría de lechones apenas se integra (10-15\%), el engorde puede someterse a este régimen contractual entre el 65 y el $75 \%$ del total.

Las cifras globales de integración de broiler, según los datos del Ministerio de Economía y Hacienda, enmascaran las diferencias existentes entre Valencia y Castellón ${ }^{10}$. Si bien las dos provincias van a la par y superan el 90\% de explotaciones integradas (Castellón alcanza el 98'6\%), no sucede lo mismo con los animales, pues mientras en Castellón casi es absoluto el grado de integración (98'9\%), en Valencia dicho porcentaje sólo representa el 76'4\%. Esto se debe al papel decisivo que desempeña la empresa alcireña independiente Luis Suñer, S. A. (Avidesa) con más de 1'5 millones de pollos cebados durante el primer semestre de 1988, lo que distorsiona el porcentaje de integración provincial (vid. figs. 1 y 2).

La producción valenciana de pollos en integración (que es lo mismo que hablar del censo avícola de carne) se ubica con preferencia en áreas situadas a menos de 400metros de altitud por la merma que supone la calefacción en los beneficios. Se trata de los llanos

\footnotetext{
${ }^{8}$ ALDOMA BUIXADE, J., VILLARREAL MORENO, J., VIÑAS FOLCH, Ll., op. cit., p. 85.

${ }^{9}$ GOSÁLVEZ PÉREZ, V., «La expansión de la ganadería 'integrada' en la provincia de Castellón, apoyo a una agricultura familiar en crisis», en Estructuras y regímenes de tenencia de la tierra en España, Madrid, Ministerio de Agricultura, 1987, p. 273.

${ }^{10}$ Omitimos los comentarios sobre la provincia de Alicante por la manifiesta infravaloración y escasa fiabilidad de sus datos.
} 


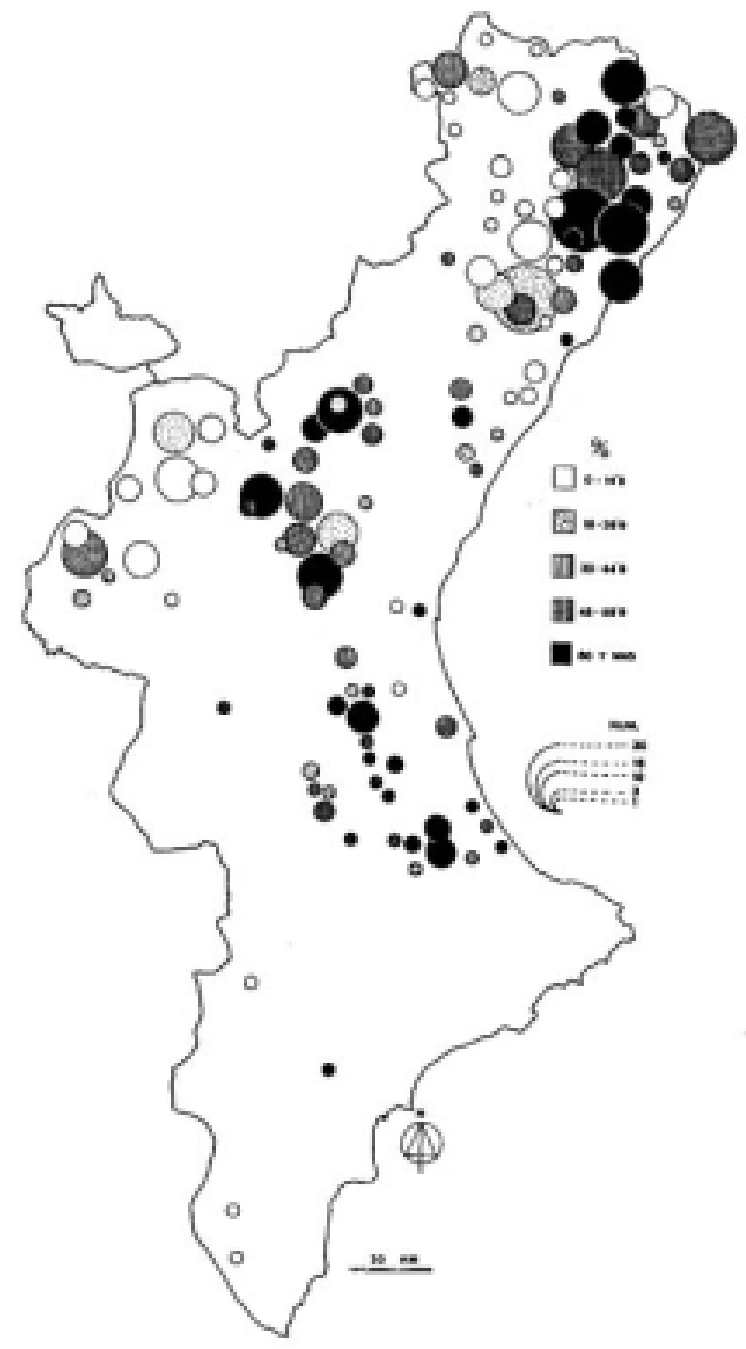

FIGURA 3. Comunidad Valenciana. Distribución municipal de las explotaciones porcinas integradas y su proporción respecto al total. Julio 1988. 


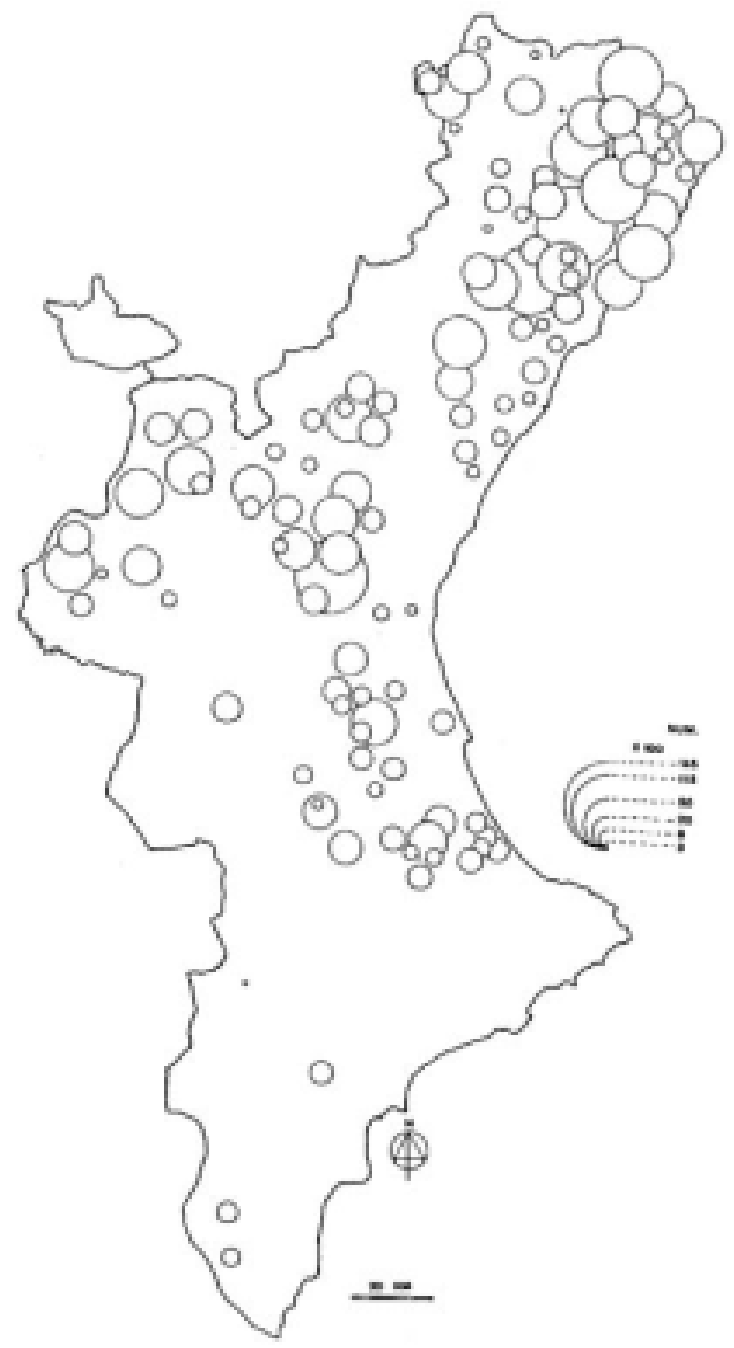

FIGURA 4. Comunidad Valenciana. Distribución municipal del ganado porcino en integración. Julio 1988. 
del litoral y prelitoral castellonense y de la provincia de Valencia (La Safor, La Vall d'Albaida, La Ribera...). Es precisamente en estas zonas de Valencia donde las explotaciones independientes son más frecuentes, pero tienen por regla general menor tamaño que las integradas y eligen una ubicación aislada, en municipios poco avícolas. Las granjas integradas suelen ser de mayores dimensiones y propician concentraciones territoriales muy acusadas. El determinismo físico, la tradición, el mimetismo y las economías de localización aglutinan la producción aviar en zonas concretas que son objeto de interés para las empresas integradoras. De esta forma se consiguen verdaderos núcleos de integración como Pinet (23 granjas y 730.000 pollos integrados durante el primer semestre de 1988), Barig (18 y 767.000), Luchente (14 y 418.000), Cuatretonda (7 y 495.000) o Carlet (7 y 303.000) entre otros. Este fenómeno está mucho más desarrollado y extendido territorialmente en Castellón, donde, por ejemplo, la Vall d'Alba ofrece 51 granjas y 1.148.000 broiler integrados para el mismo período. Otros municipios también ofrecen cifras importantes: Cuevas de Vinromá (29 y 950.000), Adzaneta (23 y 390.000), La Jana (21 y 622.000), Traiguera (20 y 727.000), Vinaroz (17 y 481.000), Alcalá de Chivert (15 y 527.000), Salsadella (12 y 330.000), etc. Sin embargo, las granjas castellonenses exhiben menores dimensiones medias que las valencianas, lo cual denota que las formidables concentraciones de animales integrados se consiguen por la proliferación del número de instalaciones y no debido a empresas muy capaces. Ello es fruto de la intensa labor integradora de varias empresas autóctonas y foráneas que copan progresivamente la totalidad de la producción. Concentran su actividad en comarcas concretas y con carácter más acusado en determinados municipios que ofrecen altos niveles de congestión. Asimismo, la actividad integradora castellonense es más antigua que la valenciana por su cercanía a Cataluña (vid. figs. 3 y 4$)$.

La integración valenciana del ganado porcino se concentra con prioridad en las explotaciones de relativo tamaño y en las que se orientan al cebo. Las empresas integradoras buscan ante todo rentabilidad, beneficios y nulas dificultades técnicas y de manejo, de ahí que centren su actividad en las granjas de engorde con dimensiones óptimas. Por estas razones, el grado de integración es bajo en las zonas de minifundio, donde además predomina la producción de lechones, es decir, el Bajo Segura, Baix Vinalopó, el interior valenciano (sobre todo Los Serranos) y algo menos en las Huertas y el sector central de Castellón, pero con manifiesta tendencia hacia el área montañosa noroccidental(Els Ports, L'Alcalatén, Alt Maestrat). La producción integrada destaca en Castellón, de preferente orientación al cebo, sobre todo en las comarcas litorales y prelitorales y a mayor distancia el área que centra Jérica. Las principales aglomeraciones municipales se dan en la Vall d'Alba (30 granjas y 10.100 cerdos integrados en 1988), Cuevas de Vinromá (26 y 14.500), Salsadella (18 y 9.800), Vinaroz (17 y 4.500), Rosell (14 y 9.400), Jérica (14 y 4.700), San Mateo (12 y 7.000), etc.

La provincia de Valencia tiene las áreas integradas más densas en el Camp del Túria y algunos sectores de La Ribera y La Safor, aunque también existen muchas zonas minifundistas y orientadas a la producción que contemplan la penetración de grandes cebaderos integrados (Los Serranos o Requena-Utiel), unidades productivas que si en términos relativos suponen una pequeña parte del total de explotación (por debajo del 30\% y en ocasiones del 15\%) en términos absolutos ofrecen cifras notables. Los valores más elevados se logran en Villamarchante (14 granjas y 11.400 animales integrados en 1988), Fuenterrobles (14 y 5.700), Villar del Arzobispo (12 y 4.100), Tuéjar (14 y 5.200), Titaguas (11 y 2.000), Lliria (11 y 4.500), Utiel (8 y 3.100$)$, Pedralba (7 y 3.600), Carlet

(7 y 5.000), etc.

En definitiva, el inseparable binomio ganadería intensiva-ganadería integrada per- 
mite el auge pecuario de áreas peninsulares poco aptas físicamente para este aprovechamiento. Es el caso de la Comunidad Valenciana, donde las modernas granjas industrializadas de cerdos y broiler han sustituido a los animales de corral y a los rebaños extensivos de ganado ovino y caprino. Las nuevas técnicas jurídico-productivas encuentran fuerte demanda en los secanos deprimidos, lugares de cultivos poco rentables que necesitan incrementar las rentas de la explotación familiar.

\section{Las empresas integradoras}

En la Comunidad Valenciana se superpone la acción integradora de varias empresas que contribuyen a modificar los sistemas ganaderos tradicionales y a extender el modelo intensivo e integrado por vastas zonas. A su vez, el crecimiento pecuario atrae nuevas empresas y genera diversas áreas de influencia. Aquí operan desde poderosas multinacionales (Piensos Hens, Nanta, Conagra...) para las que las tierras valencianas sólo son un sector más dentro de su estrategia nacional hasta empresas de raigambre local (La Florida, Casa Roc, Vigorán...), pasando por otras, bien autóctonas bien extranjeras, que desde Navarra (Uvesa), Aragón (Gil Albesa) y sobre todo Cataluña (Vall Companys, Fabra, Dertosa, Guissona...) penetran en la Comunidad Valenciana como consecuencia de su política expansionista Asimismo, encontramos una constelación de pequeños integradores extraordinariamente coyunturales que, al igual que en Cataluña, fluctúan según las oscilaciones del mercado e intentan conseguir a corto plazo gran rentabilidad de las inversiones que realizan integrando pocas y modestas explotaciones locales ${ }^{11}$. Estas integradoras pequeñas y de talante familiar son más abundantes en la actividad porcina porque la rentabilidad unitaria es mayor que en las aves.

Las firmas multinacionales son las que gozan de mayor peso específico. Tienen una difusión territorial acusada en consonancia con una filosofía económica de planteamientos nacionales, ya que su acción sobre la ganadería a través de integraciones, fabricación de piensos compuestos, industrias cárnicas, comercialización de productos, etc., afecta a gran parte del país. Por ejemplo, Piensos Hens (filial de Cargill-EE.UU.) posee fábricas de piensos en Cataluña, Valencia, Zaragoza, Andalucía, León, Baleares y Murcia, mientras que las de Nanta (filial de Hendrix-Países Bajos) se reparten por Cataluña, Madrid, Canarias, Murcia, Extremadura, Castilla-León y Valencia. Tanto una como otra actúan en lugares de ganadería intensiva boyante aunque se percibe el intento de Nanta de penetrar en nuevas áreas.

Las empresas autóctonas son más modestas en sus actuaciones aunque cuenten con fábricas de piensos, redes de comercialización, mataderos y radios de influencia más o menos amplios. Algunas de ellas gozan de notable representación en el proceso integrador regional.

En cualquier caso, la influencia territorial de las principales firmas integradoras sigue, con ligeros matices, pautas semejantes. Estas empresas prefieren, una vez presentes en una zona, consolidar su posición absorbiendo el mayor número posible de ganaderos antes que iniciar el proceso integrador en áreas distintas y/o escasamente pecuarias. La mayoría de los integradores, si no tienen carácter local o comarcal, suelen concentrarse, grosso modo, en los mismos sectores, allí donde la ganadería intensiva es de reciente implanta-

${ }^{11}$ ALDOMA BUIXADE, J., VILLARREAL MORENO, J., «Integración: fenómeno decisivo en el desarrollo ganadero», El Campo, 95, julio-septiembre 1984, p. 139. 


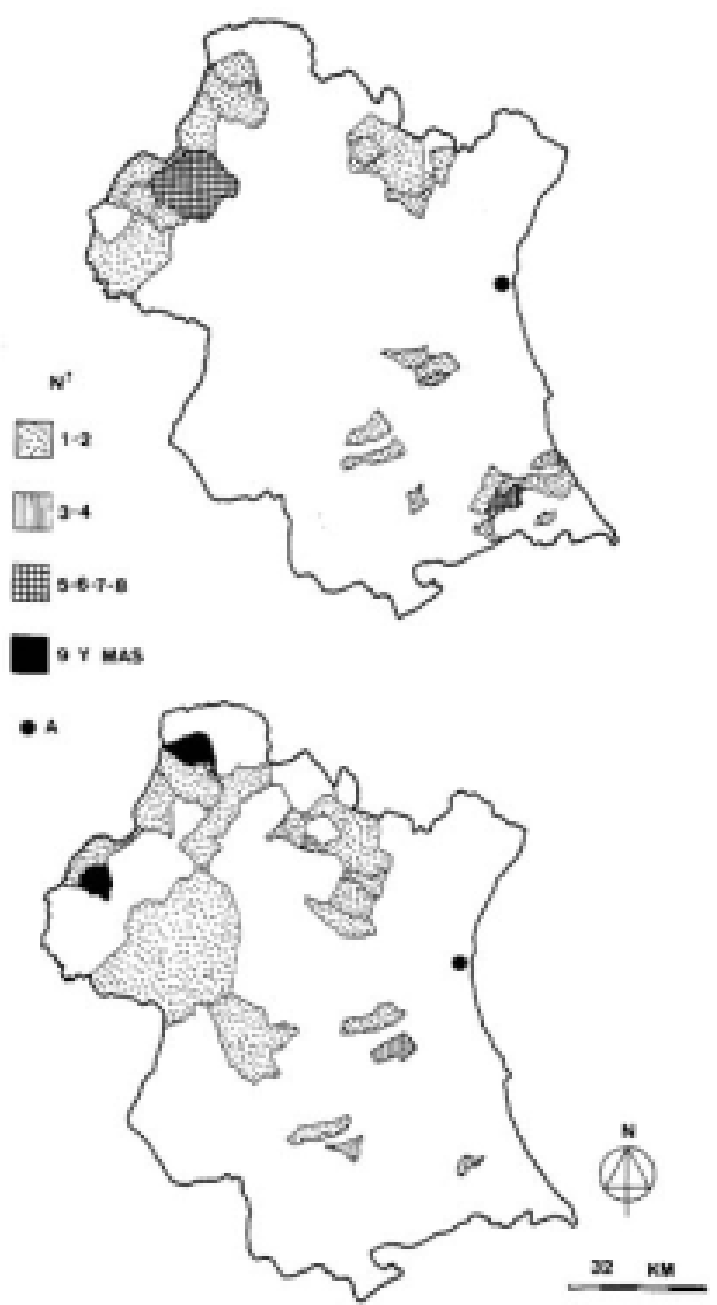

FIGURA 5. Número de explotaciones porcinas integradas por Piensos Hens (mapa superior) y por Nanta (mapa inferior) en los municipios de la provincia de Valencia. Julio 1988. A, Lugar donde radica la fábrica de piensos de la empresa integradora. 


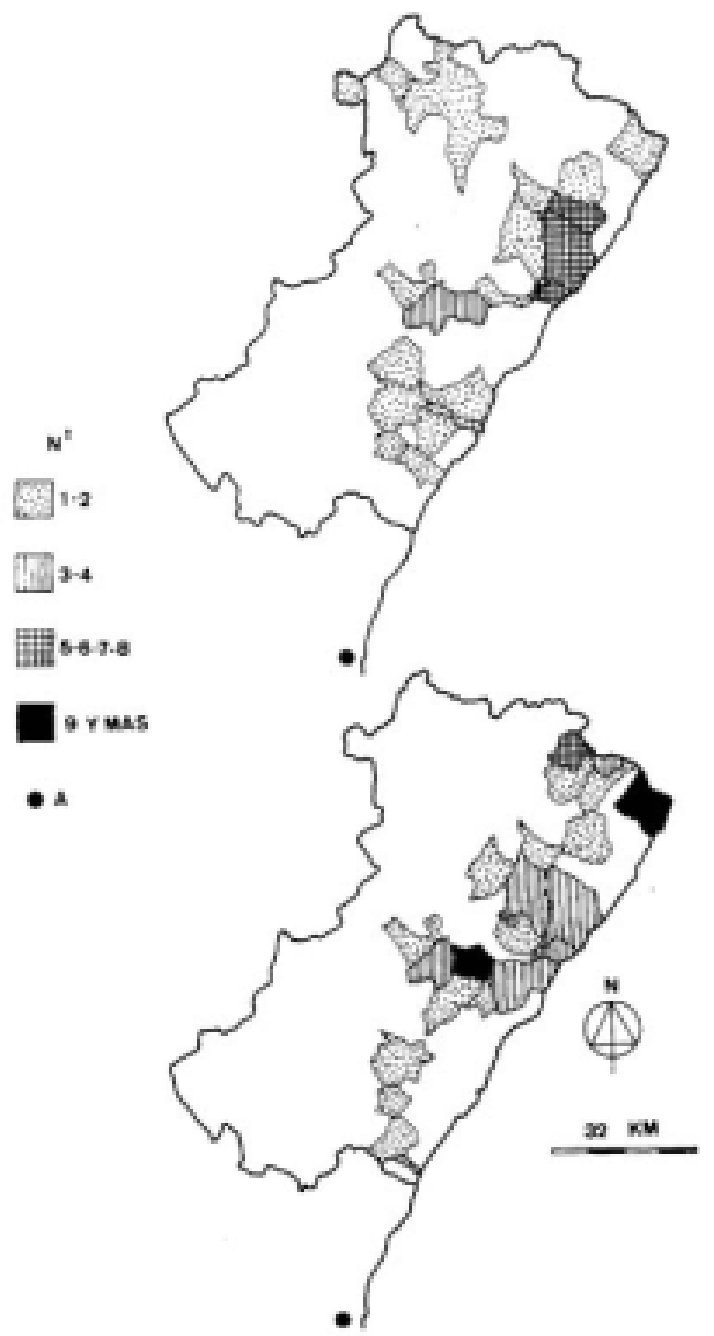

FIGURA 6. Número de explotaciones porcinas integradas por Piensos Hens (mapa superior) y por Nanta (mapa inferior) en los municipios de la provincia de Castellón. Julio 1988. A, Lugar donde radica la fábrica de piensos de la empresa integradora. 
ción, la agricultura es muy poco rentable y existe un excelente caldo de cultivo porque los granjeros no pueden o no quieren asumir los riesgos del mercado. En estos lugares, compiten las empresas más agresivas y luchan por conseguir mayor representatividad. Suelen coincidir en las mismas comarcas pero se dan sensibles diferencias en cuanto a la entidad que cada una tiene en los distintos municipios, incluso alguno de ellos queda vacío de la acción de empresas concretas porque ya ha penetrado otra con anterioridad. En términos municipales muy densos, con abundantes granjas (v. gr. la Vall d'Alba), se acumula el influjo de varias integradoras que generan un dinámico proceso productivo-comercial.

Un factor de enorme importancia en la construcción de las áreas de influencia de las empresas integradoras es la proximidad y/o las buenas comunicaciones. El principal objetivo es abaratar los costes de transporte y para ello intentan que la mayor parte de los elementos que intervienen en la producción se ubiquen lo más cerca posible de la fábrica de piensos o que dispongan de un acceso fácil y directo por carretera. No olvidemos que la propia intensidad productiva que define los modernos métodos de producción lleva consigo estrechos y continuos contactos. Los intercambios de input y output son constantes.

Las empresas multinacionales se extienden con profusión por Castellón y Valencia, erigiendo dilatadas redes de influencia espacial. Desde sus fábricas de piensos situadas en los municipios que rodean a la ciudad de Valencia proyectan su influjo hasta los confines de la región (vid. figs. 5 y 6). A partir del núcleo de la cadena productiva (fábrica de piensos) acceden a sus granjas integradas a través del contacto que propician ciertos ejes viarios. En Valencia, las carreteras que facilitan este tránsito son la N-III que une el litoral con la zona de Requena-Utiel y la C-234 que alcanza Los Serranos después de cruzar el Camp del Túria, es decir, los principales sectores porcinos. Los núcleos avícolas meridionales utilizan la N-340, pero aquí el factor proximidad es más determinante por los cortos ciclos de engorde de los broiler. En la provincia de Castellón, la actividad integradora se concentra a lo largo de la N-340, paralela a la costa, y de la C-238 que con múltiples ramificaciones locales cruza los llanos litorales en sentido N-S.

Las empresas autóctonas suelen tener un radio de acción más moderado. Por ejemplo, las catalanas con sede en Tortosa y Amposta no van más allá de los municipios castellonenses limítrofes con Tarragona (vid. figs. 7 y 8). Las firmas locales se circunscriben por regla general a los municipios de su comarca (v. gr. Casa Roc. Fig. 8), pero merece la pena destacar el papel regional que asume La Florida, S. A. Esta empresa, radicada en Vila-real, extiende sus integraciones por Castellón y Valencia; sin embargo, son los municipios de sus proximidades los que contemplan una acción más decidida y los que albergan mayor número de granjas en integración (vid. fig. 7).

\section{Consecuencias del fenómeno integrador}

El fenómeno integrador ha tenido repercusiones positivas. Esta fórmula permite extender la actividad pecuaria intensiva en áreas poco ganaderas y estimula el crecimiento de la producción cárnica de aves y porcino con el consiguiente abaratamiento de los precios para el consumidor. Propicia también la introducción de tecnología moderna y criterios empresariales para lograr gran productividad, así como una mejora en los canales de comercialización. Por otro lado, tolera la permanencia del ganadero en el sector y en el medio rural al tiempo que éste evita los riesgos propios del mercado. Muchos agricultores de zonas deprimidas pueden incrementar sus rentas con la instalación de granjas 


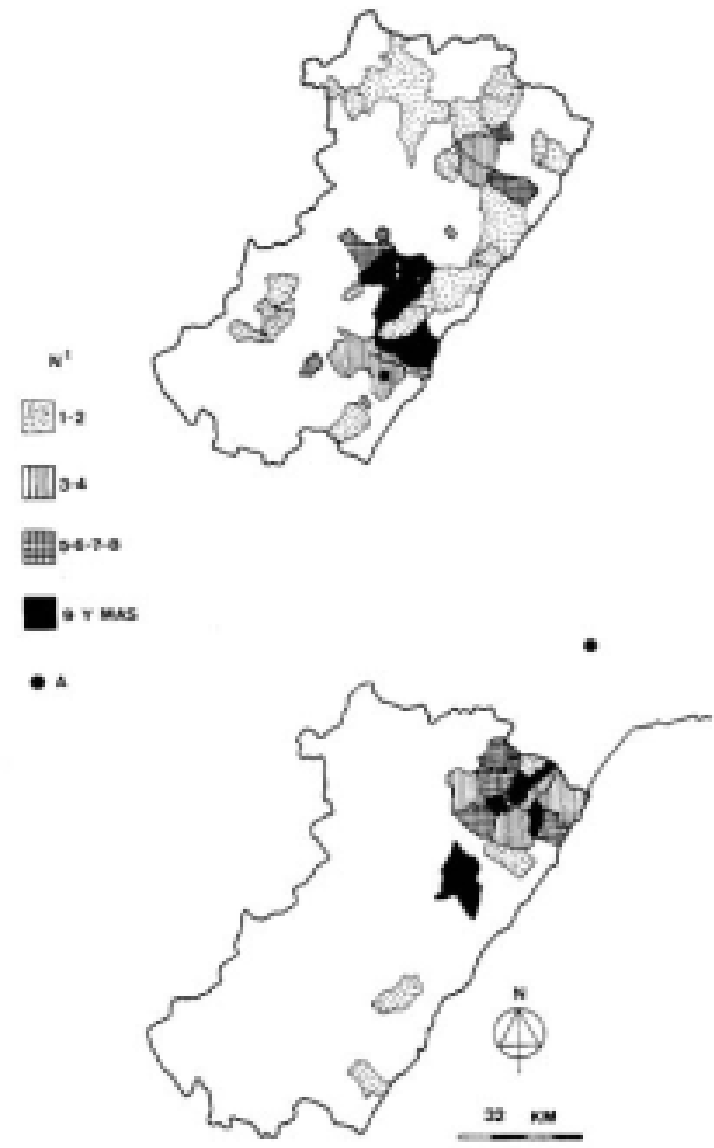

FIGURA 7. Número de explotaciones avícolas de carne integradas por La Florida (mapa superior) y por Fabra (mapa inferior) en los municipios de la provincia de Castellón. Julio 1988. A, Lugar donde radica la fábrica de piensos de la empresa integradora. 

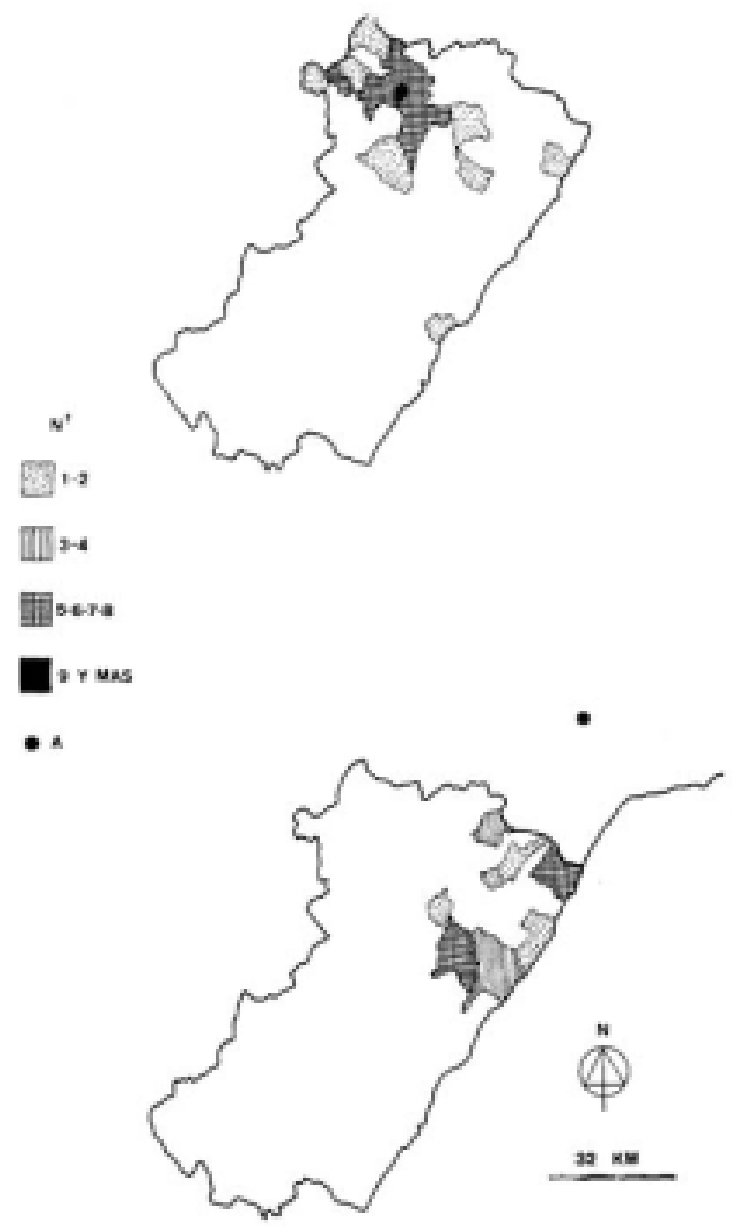

FIGURA 8. Número de explotaciones porcinas integradas por Casa Roc (mapa superior) y por Dertosa (mapa inferior) en los municipios de la provincia de Castellón. Julio 1988. A, Lugar donde radica la fábrica de piensos de la empresa integradora. 
que les posibilita dedicación pecuaria a tiempo parcial. Sin demasiadas horas de trabajo se generan ingresos que en ocasiones son notables.

Estas incuestionables ventajas no deben ensombrecer una serie de graves realidades. Al principio, los granjeros compraban el pienso, los medicamentos y los animales a las fábricas de concentrados, siendo muy fuertes las conexiones entre éstas y las multinacionales que dominan las materias primas y la genética. El granjero era propietario del ganado y de los input, gestionaba su explotación y asumía los riesgos del mercado. Incluso la financiación de las instalaciones corría a cargo de la fábrica de piensos. Sin embargo, conforme pasa el tiempo y las sucesivas crisis hacen mella en las explotaciones menos capitalizadas, las grandes empresas comienzan a dominar la producción e imponen contratos de arrendamiento de servicio, es decir, la integración tal y como la conocemos en la actualidad. La empresa integradora es propietaria de los animales, piensos y productos zoosanitarios y se los proporciona, junto con la asistencia técnica y veterinaria, al ganadero. Este se limita a aportar las instalaciones y la mano de obra, pero ya no posee los medios de producción. En realidad es como si el integrado «alquilara» la granja al integrador, pierde autonomía y capacidad de decisión y no asume riesgos.

Esta posición preeminente y dominadora permite a la integradora imponer sus propias condiciones, lo cual se ve favorecido por la inexistencia de aparato legislativo. La ganadería integrada se halla presente en España desde los años sesenta y sin embargo no está regulada por ninguna normativa legal. Solamente la Generalitat de Catalunya ha legislado sobre este aspecto (Llei 24/1984, de 28 de novembre-DOGC 14-desembre-1984 y Decret 54/1985, de 18 de febrer-DOGC 15-març-1985), aunque la inscripción en el Registre de Contractes d'Integració no es obligatoria y ello suscitó en su momento una viva polémica junto con la demanda de mayor transparencia en las relaciones contractuales ${ }^{12}$. Además, es necesaria una legislación estatal para evitar que los integradores catalanes se expanden por otras regiones en las mismas condiciones de dominio.

Ante semejante vacío legal, las relaciones entre integrado e integrador se reducen a contratos-tipo, no homologados, en los que el ganadero no tiene ningún poder de decisión. Estos contratos, confeccionados por cada empresa integradora, suelen ofrecer características comunes, aunque con frecuencia introduzcan matices definitorios de los rasgos de las diversas regiones $o$ comarcas del país e incluso de cada firma. Muchas empresas introducen unilateralmente en los contratos matices, en ocasiones muy ambiguos, cuyo único norte es la acumulación de capital sin tener en cuenta la enorme y progresiva presión que ejercen sobre el ganadero. Este se ve obligado a aceptar cualquier cláusula adoptando una postura conformista si quiere permanecer en el sector y seguir contratado por la integradora. El simple análisis de los contratos utilizados por ciertas empresas, tanto para porcino como para pollos, es suficiente ejemplo del desequilibrio imperante en el conjunto de derechos y obligaciones que contraen las dos partes. Cualquier minucia basta para que el ganadero no perciba, en todo o en parte, las primas de producción prefijadas.

Por su parte, la Administración ha permanecido mucho tiempo impasible pese a las reivindicaciones de varios colectivos sindicales, sobre todo la Coordinadora de Organizaciones de Agricultores y Ganaderos (COAG). Como ejemplo baste citar el tema de la Licencia Fiscal, de especiales repercusiones en la provincia de Valencia. El Ministerio de Economía y Hacienda considera industriales a los ganaderos integrados y ganaderos a

\footnotetext{
${ }^{12}$ Diario El País, Madrid, 8-enero-1983. Diario La Mañana, Lleida, 8-enero-1983.
} 
las empresas integradoras. Mientras a los segundos les aplica el Régimen Especial Agrícola y Ganadero, a los primeros les exige la Licencia Fiscal de Actividades Comerciales e Industriales. Ello afecta incluso a cuestiones como la de la Seguridad Social, ya que el impuesto industrial aleja a los ganaderos de todo régimen agrario.

En este contexto, el integrador puede generar beneficios en muy poco tiempo, no necesita acometer inversiones cuantiosas, reduce los gastos al mínimo, no paga Seguridad Social, elimina los problemas laborales, concentra gran poder económico y controla los precios, producciones y mercados. Por el contrario, el ganadero se inserta en el proceso integrador impelido por circunstancias desfavorables de carácter coyuntural y/o estructural ajenas a su voluntad, su única opción es integrarse o desaparecer. A cambio de soslayar riesgos y continuar en la actividad que le proporciona ingresos complementarios, cede su capacidad de gestión y autonomía y su influencia en el balance oferta-demanda; cada vez es más presionado en aras de la maximación de beneficios, la acumulación de capital y la rentabilidad. La integración supone entonces una «matizada y sutil forma de proletarización» ${ }^{13}$.

Muchos grandes integradores justifican el fenómeno integrador con la necesidad de mantener el consumo cárnico. Por lo tanto, según ellos, la explotación familiar no es compatible con los métodos intensivos que satisfacen a precios asequibles las demandas poblacionales. La infraestructura que requiere la industrialización pecuaria no puede conseguirse individualmente por su alto coste. De esta forma se explica el desarrollo de capitalizados emporios empresariales y de las integraciones que practican. Quizás la solución transitoria, que atenúe estas desiguales relaciones, teniendo en cuenta el actual sistema económico y ante las oscuras perspectivas que asolan la producción ganadera familiar, radique en el modelo catalán de cooperativas basado en la autointegración, donde el integrado es socio y participa en las decisiones y en los beneficios.

${ }^{13}$ GÁMIZ LÓPEZ, A., «Agricultura familiar y dependencia en la producción bajo contrato», Agricultura y Sociedad, 1, octubre-diciembre 1976, p. 78. 